\title{
Observations of GRBs in the mm/submm range at the dawn of the ALMA era
}

\author{
A. de Ugarte Postigo ${ }^{1,2}$, A. Lundgren ${ }^{3,4}$, S. Martín ${ }^{3}$, \\ D. García-Appadoo ${ }^{3,4}$, I. de Gregorio Monsalvo ${ }^{3,4}$, C.C. Thöne ${ }^{1}$, \\ J. Gorosabel ${ }^{1}$, A. J. Castro-Tirado ${ }^{1}$, R. Sánchez-Ramírez ${ }^{1}$, \\ and J. C. Tello ${ }^{1}$ on behalf of a larger collaboration \\ ${ }^{1}$ Instituto de Astrofísica de Andalucía (IAA-CSIC), Spain; \\ email: deugarte@iaa.es \\ ${ }^{2}$ Dark Cosmology Centre, Niels Bohr Institute, Univ. of Copenhagen, Denmark ${ }^{3}$ European \\ Southern Observatory, Chile ${ }^{4}$ Joint ALMA Observatory, Chile
}

\begin{abstract}
Gamma-ray bursts (GRBs) generate an afterglow with an emission peaking in the millimetre and submillimeter $(\mathrm{mm} / \mathrm{submm})$ range during the first hours to days, making the study in these wavelengths of great importance. Here we give an overview of the data that has been collected for GRB observations in this wavelengths until September 2011. The total sample includes 102 GRBs, of which 88 have afterglow observations, and the rest are only host galaxy searches. The 22 detections cover the redshift range between 0.168 and 8.2 and have peak luminosities that span 2.5 orders of magnitude. With the start of the operations at ALMA, the sensitivity with respect to previous facilities has already improved by over an order of magnitude. We estimate that, once completed, ALMA will be able to detect $\sim 98 \%$ of the afterglows.
\end{abstract}

This proceeding is based on the work published by de Ugarte Postigo et al. (2012).

Keywords. gamma rays: bursts, submillimeter

\section{Observations and sample}

During the last 3 years we have followed-up 11 GRBs (plus an additional Galactic X-ray binary, initially identified as a GRB) and discovered 2 counterparts in submm with observing programmes at APEX and SMA. This is put into context with the most complete sample of continuum observations that have been published to date of GRB afterglows and their host galaxies in the $\mathrm{mm} / \mathrm{submm}$ wavelength range, covering from early 1997 until the 30th of September 2011.

The complete sample includes observations of 102 bursts, of which 88 are searches for GRB afterglows, with 22 detections. There have been specific host galaxy searches for 36 cases, although limits can be provided for the 102 bursts that have been followed. Host galaxy detections have only been achieved in four cases: GRB 000210, GRB 000418, GRB 010222 and XT 080109.

\section{Afterglow models}

GRB afterglows can be described, in the simplest case, using the fireball model (Sari et al. 1998). According to it, material is ejected at ultrarelativistic velocities through collimated jets. When this material interacts with the medium surrounding the progenitor, the accelerated particles emit a synchrotron spectrum that is characterised by three break frequencies: $\nu_{m}$ is the characteristic synchrotron frequency and is the maximum 
of the emission, $\nu_{c}$ is the cooling frequency, above which radiative cooling is significant, and $\nu_{a}$ is the synchrotron self-absorption frequency.

A reverse shock, produced inside the ejecta, can generate an additional early emission (Piran et al. 1999). This has been rarely observed in the optical wavelengths but is expected to have a significant contribution in the early $\mathrm{mm} / \mathrm{submm}$ emission. For example, the mm detection of GRB 090423, at a redshift of 8.2, seems to show excess emission possibly due to a reverse shock.

\section{Observations of individual GRBs}

Multiwavelength observations of GRB afterglows are the only way to determine with precision the physics involved in the GRB and learn about the environment that surrounds it. Studies in $\mathrm{mm} / \mathrm{submm}$ are especially interesting in the case of optically-dark bursts, as they allow us to access what optical observations cannot.

Optical samples of GRBs are limited by the extinction in the host galaxy that, if large, make the optical emission undetectable. The negligible effect of dust extinction in the $\mathrm{mm} / \mathrm{submm}$ bands allows us to study a more complete sample. As an example we can look at GRB 051022, one of the darkest bursts detected to date, for which an optical counterpart was not found. Observations in mm wavelengths allowed us to localise and study the afterglow and host galaxy (Castro-Tirado et al. 2007). The other main cause for optically dark GRBs is their high redshift. In these cases the absorption produced at frequencies higher than the Lyman limit does not allow us to obtain optical detections of GRB afterglows beyond redshifts of 6 . These events are important to understand the formation of the first stars in the Universe. Proof that they can be detected in the $\mathrm{mm} /$ submm range is the fact that, out of the three GRB afterglows observed at $\mathrm{z}>6$, two have been detected (GRB 050904 at $\mathrm{z}=6.3$, Tagliaferri et al. 2005, Haislip et al 2006, and GRB 090423 at $\mathrm{z}=8.2$, Tanvir et al. 2009, Salvaterra et al. 2009).

\section{GRBs in the ALMA era}

Using data from the sample and assumptions based on samples at other wavelengths, we estimate the real peak flux density distribution of GRBs, from which an average peak flux density value of $0.33 \mathrm{mJy}$ can be expected. Using the detection limits calculated for ALMA, we can expect that the completed observatory should be able to detect $98 \%$. In the case of bright GRB afterglows, ALMA will be able to study spectral features and perform polarimetric studies of the afterglow, which have been out of reach until now. With ALMA we will be, for the first time, in position to undertake studies of samples of GRB host galaxies. We will be able to perform studies of the continuum emission to characterise the dust content and determine the unextinguished star formation rate of the hosts. Through the study of emission features from the host, we will be able to understand the molecular content and the chemical enrichment of the strong star-forming regions in which GRBs are found, at redshifts that go back to the epoch in which the first stars were formed.

\section{References}

Castro-Tirado, A. J., Bremer, M., McBreen, S., et al. 2007, A\&̈A, 475, 101

Haislip, J. B., Nysewander, M. C., Reichart, D. E., et al. 2006, Nature, 440, 181

Piran, T. 1999, Physics Reports, 314, 575 
Salvaterra, R., Della Valle, M., Campana, S., et al. 2009, Nature, 461, 1258

Sari, R., Piran, T., \& Narayan, R. 1998, ApJL, 497, L17

Tagliaferri, G., Antonelli, L. A., Chincarini, G., et al. 2005, A\&A, 443, L1

Tanvir, N. R., Fox, D. B., Levan, A. J., et al. 2009, Nature, 461, 1254

de Ugarte Postigo, A., Lundgren, A., Martín, S., et al. 2012, A\&3A, 538, A44 